\title{
Remarks on Blow-Up Phenomena in $p$-Laplacian Heat Equation with Inhomogeneous Nonlinearity
}

\author{
ALZAHRANI Eadah Ahma and MAJDOUB Mohamed* \\ Deapartment of Mathematics, College of Science, Imam Abdulrahman Bin Faisal \\ University, P. O. Box 1982, Dammam, Saudi Arabia E Basic and Applied Scientific \\ Research Center, Imam Abdulrahman Bin Faisal University, P.O. Box 1982, 31441, \\ Dammam, Saudi Arabia.
}

Received 24 June 2020; Accepted 29 August 2020

\begin{abstract}
We investigate the $p$-Laplace heat equation $u_{t}-\Delta_{p} u=\zeta(t) f(u)$ in a bounded smooth domain. Using differential-inequality arguments, we prove blow-up results under suitable conditions on $\zeta, f$, and the initial datum $u_{0}$. We also give an upper bound for the blow-up time in each case.
\end{abstract}

AMS Subject Classifications: 35K55, 35K65, 35K61, 35B30, 35B44

Chinese Library Classifications: O175.26

Key Words: Parabolic problems; p-Laplacian equation; blow-up; positive initial energy.

\section{Introduction}

In the past decade a strong interest in the phenomenon of blow-up of solutions to various classes of nonlinear parabolic problems has been assiduously investigated. We refer the reader to the books $[1,2]$ as well as to the survey paper [3]. Problems with constant coefficients were investigated in [4], and problems with time-dependent coefficients under homogeneous Dirichlet boundary conditions were treated in [5]. See also [6] for a related system. The question of blow-up for nonnegative classical solutions of $p$-Laplacian heat equations with various boundary conditions has attracted considerable attention in the mathematical community in recent years. See for instance [7-10].

There are two effective techniques which have been employed to prove non-existence of global solutions: the concavity method ([11]) and the eigenfunction method ([12]). The

${ }^{*}$ Corresponding author. Email addresses: ealzahrani@iau.edu.sa (E. A. Alzahrani), mmajdoub@iau.edu.sa (M. Majdoub) 
latter one was first used for bounded domains but it can be adapted to the whole space $\mathbb{R}^{N}$. The concavity method and its variants were used in the study of many nonlinear evolution partial differential equations (see, e.g., [13-15]).

In the present paper, we investigate the blow-up phenomena of solutions to the following nonlinear $p$-Laplacian heat equation:

$$
\begin{cases}u_{t}-\Delta_{p} u=\zeta(t) f(u), & x \in \Omega, \quad t>0, \\ u(t, x)=0, & x \in \partial \Omega, \quad t>0 \\ u(0, x)=u_{0}(x), & x \in \Omega,\end{cases}
$$

where $\Delta_{p} u:=\operatorname{div}\left(|\nabla u|^{p-2} \nabla u\right)$ is the $p$-Laplacian operator, $p \geq 2, \Omega$ is a bounded sufficiently smooth domain in $\mathbb{R}^{N}, \zeta(t)$ is a nonnegative continuous function. The nonlinearity $f(u)$ is assumed to be continuous with $f(0)=0$. More specific assumptions on $f, \zeta$ and $u_{0}$ will be made later.

The case of $p=2$ has been studied in [4] for $\zeta(t) \equiv 1$, and in [5] for $\zeta$ being a nonconstant function of $t$. Concerning the case $p>2$, Messaoudi [10] proved the blow-up of solutions with vanishing initial energy when $\zeta(t) \equiv 1$. See also [9] and references therein. Recently, a $p$-Laplacian heat equations with nonlinear boundary conditions and timedependent coefficient was investigated in [7]. This note may be regarded as a complement, and in some sense an improvement, of $[5,10]$.

Let us now precise the assumptions on $f$ and $\zeta$. If $p=2$, we suppose either

$$
\begin{aligned}
& f \in C^{1}(\mathbb{R}) \quad \text { is convex with } f(0)=0 ; \\
& \exists \lambda>0 \text { such that } f(s)>0 \text { for all } s \geq \lambda ; \\
& \int_{\lambda}^{\infty} \frac{\mathrm{d} s}{f(s)}<\infty ; \\
& \inf _{t \geq 0}\left(\int_{0}^{t}(\zeta(s)-1) \mathrm{d} s\right):=m \in(-\infty, 0],
\end{aligned}
$$

or

$$
s f(s) \geq(2+\epsilon) F(s) \geq C_{0}|s|^{\alpha},
$$

for some constants $\epsilon, C_{0}>0, \alpha>2$, and

$$
\zeta \in C^{1}([0, \infty)) \text { with } \zeta(0)>0 \text { and } \zeta^{\prime} \geq 0 .
$$

Here $F(s)=\int_{0}^{s} f(\tau) \mathrm{d} \tau$.

Our first main result concerns the case $p=2$ and reads as follows.

Theorem 1.1. Suppose that assumptions (1.2)-(1.5) are fulfilled. Let $0 \leq u_{0} \in L^{\infty}(\Omega)$ such that $\int_{\Omega} u_{0} \phi_{1}$ is large enough. Then the solution $u(t, x)$ of problem (1.1) blows up in finite time. 


\section{Remark 1.1.}

(i) The function $\phi_{1}$ stands for the eigenfunction of the Dirichlet-Laplace operator associated to the first eigenvalue $\lambda_{1}>0$, that is

$$
\Delta \phi_{1}=-\lambda_{1} \phi_{1}, \quad \phi_{1}>0, x \in \Omega ; \quad \phi_{1}=0, x \in \partial \Omega, \int_{\Omega} \phi_{1}=1 .
$$

(ii) The assumptions (1.2)-(1.5) on $f$ and $\zeta$ cover the example

$$
f(u)=\mathrm{e}^{u}-1 \quad \text { and } \quad \zeta(t)=\mathrm{e}^{t^{2}} .
$$

Note that this example is not studied in [5], and Theorem 1.1 can be seen as an improvement of Theorem 1 of [5].

(iii) As it will be clear in the proof below, an upper bound of the maximal time of existence is given by

$$
T^{*}=-m+2 \int_{y_{0}}^{\infty} \frac{\mathrm{d} s}{f(s)},
$$

where $m$ is as in (1.5) and $y_{0}=\mathrm{e}^{m \lambda_{1}} \int_{\Omega} u_{0} \phi_{1}$.

(iv) The conclusion of Theorem 1.1 remains valid for $\Omega=\mathbb{R}^{N}$ if we replace $\phi_{1}$ by $\varphi(x)=$ $\pi^{-N / 2} \mathrm{e}^{-|x|^{2}}$

In order to state our next result (again for $p=2$ ), we introduce the energy functional

$$
E(u(t)):=\frac{1}{2} \int_{\Omega}|\nabla u(t, x)|^{2} \mathrm{~d} x-\zeta(t) \int_{\Omega} F(u(t, x)) \mathrm{d} x .
$$

Using (1.7), we see that $t \longmapsto E(u(t))$ is nonincreasing along any solution of (1.1). This leads to the following.

Theorem 1.2. Suppose that assumptions (1.6)-(1.7) are fulfilled. Assume that either $E\left(u_{0}\right) \leq 0$ or $E\left(u_{0}\right)>0$ and $\left\|u_{0}\right\|_{2}$ is large enough. Then the corresponding solution $u(t, x)$ blows up in finite time.

Remark 1.2. An upper bound for the blow-up time is given by

$$
T^{*}= \begin{cases}\frac{(2+\epsilon)|\Omega|^{\alpha / 2-1}\left\|u_{0}\right\|_{2}^{2-\alpha}}{\epsilon \zeta(0) C_{0}(\alpha-2)} & \text { if } E\left(u_{0}\right) \leq 0, \\ \int_{\left\|u_{0}\right\|_{2}^{2} / 2}^{\infty} \frac{\mathrm{d} z}{A z^{\alpha / 2}-2 E\left(u_{0}\right)} & \text { if } E\left(u_{0}\right)>0\end{cases}
$$

where

$$
A=\frac{2^{\alpha / 2} C_{0} \epsilon \zeta(0)}{(2+\epsilon)|\Omega|^{\alpha / 2-1}} .
$$


We turn now to the case $p>2$. In [16], the author studied (1.1) when $\zeta(t) \equiv 1$. He established:

- local existence when $f \in C^{1}(\mathbb{R})$;

- global existence when $u f(u) \lesssim|u|^{q}$ for some $q<p$;

- nonglobal existence under the condition

$$
\left.\frac{1}{p} \int_{\Omega} \mid \nabla u_{0}\right)\left.\right|^{p} \mathrm{~d} x-\int_{\Omega} F\left(u_{0}\right) \mathrm{d} x<0 .
$$

Later on Messaoudi [10] improved the condition (1.12) by showing that blow-up can be obtained for vanishing initial energy. Note that by adapting the arguments used in [16], we can show a local existence result as stated below.

Theorem 1.3. Suppose $\zeta \in C([0, \infty])$ and $f \in C(\mathbb{R})$ satisfy $|f| \leq g$ for some $C^{1}$-function $g$. Then for any $u_{0} \in L^{\infty}(\Omega) \cap W_{0}^{1, p}(\Omega)$, the problem (1.1) has a local solution

$$
u \in L^{\infty}((0, T) \times \Omega) \cap L^{p}\left((0, T) ; W_{0}^{1, p}(\Omega)\right), u_{t} \in L^{2}((0, T) \times \Omega) .
$$

The energy of a solution $u$ is

$$
\mathbf{E}_{p}(u(t))=\frac{1}{p} \int_{\Omega}|\nabla u(t, x)|^{p} \mathrm{~d} x-\zeta(t) \int_{\Omega} F((u(t, x)) \mathrm{d} x .
$$

We also define the following set of initial data

$$
\mathcal{E}=\left\{u_{0} \in L^{\infty}(\Omega) \cap W_{0}^{1, p}(\Omega) ; u_{0} \neq \equiv 0 \text { and } \mathbf{E}_{p}\left(u_{0}\right) \leq 0\right\} .
$$

Our main result concerning $p>2$ ca be stated as follows.

Theorem 1.4. Suppose that assumption (1.7) is fulfilled. Let $f \in C(\mathbb{R})$ satisfy $|f| \leq g$ for some $C^{1}$-function $g$ and

$$
0 \leq \kappa F(u) \leq u f(u), \quad \kappa>p>2 .
$$

Then for any $u_{0} \in \mathcal{E}$ the solution $u(t, x)$ of $(1.1)$ given in Theorem 1.3 blows up in finite time.

Remark 1.3. Theorem 1.4 and its proof are almost the result of [10]. In fact, with $\zeta$ satisfying (1.7), it only accelerate the blow-up.

Remark 1.4. Although the proof uses the Poincaré inequality in a crucial way, we believe that a similar result can be obtained for $\Omega=\mathbb{R}^{N}$. This will be investigated in a forthcoming paper.

We stress that the set $\mathcal{E}$ is non empty as it is shown in the following proposition.

Proposition 1.1. Suppose that assumption (1.15) is fulfilled and $\zeta(0)>0$. Then $\mathcal{E} \neq \varnothing$. 


\section{Proofs}

This section is devoted to the proof of Theorems 1.1-1.2-1.4 as well as Proposition 1.1.

\subsection{Proof of Theorem 1.1}

The main idea in the proof is to define a suitable auxiliary function $y(t)$ and obtain a differential inequality leading to the blow-up. Define the function $y(t)$ as

$$
y(t)=a(t) \int_{\Omega} u(t, x) \phi_{1}(x) \mathrm{d} x,
$$

where

$$
\begin{aligned}
& a(t)=\mathrm{e}^{\lambda_{1}(m-\Theta(t))}, \\
& \Theta(t)=\int_{0}^{t}(\zeta(s)-1) \mathrm{d} s .
\end{aligned}
$$

We compute

$$
\begin{aligned}
y^{\prime}(t) & =\frac{a^{\prime}(t)}{a(t)} y(t)-\lambda_{1} y(t)+a(t) \zeta(t) \int_{\Omega} f(u(t, x)) \phi_{1}(x) \mathrm{d} x \\
& =-\lambda_{1} \zeta(t) y(t)+a(t) \zeta(t) \int_{\Omega} f(u(t, x)) \phi_{1}(x) \mathrm{d} x
\end{aligned}
$$

where we have used $a^{\prime} / a-\lambda_{1}=-\lambda_{1} \zeta$. By using (1.2) and the fact that $0 \leq a \leq 1$, we easily arrive at

$$
y^{\prime}(t) \geq \zeta(t)\left(-\lambda_{1} y(t)+f(y(t))\right)
$$

Since $f$ is convex and due to (1.4), there exists a constant $C \geq \lambda$ such that $f(s) \geq 2 \lambda_{1} s$ for all $s \geq C$. Suppose $y(0)>C$. It follows from (2.4) that, as long as $u$ exists, $y(t) \geq C$. Therefore

$$
y(t) \geq \frac{\zeta(t)}{2} f(y(t))
$$

Hence

$$
\frac{t+m}{2} \leq \frac{1}{2} \int_{0}^{t} \zeta(s) \mathrm{d} s \leq \int_{y(0)}^{\infty} \frac{\mathrm{d} s}{f(s)}<\infty .
$$

This means that the solution $u$ cannot exist globally and leads to the upper bound given by (1.9). 


\subsection{Proof of Theorem 1.2}

Let $y(t)$ be the auxiliary function defined as follows

$$
y(t)=\frac{1}{2} \int_{\Omega} u^{2}(t, x) \mathrm{d} x
$$

We compute

$$
\begin{aligned}
y^{\prime}(t) & =\int_{\Omega} u(\Delta u+\zeta(t) f(u)) \mathrm{d} x \\
& =-\int_{\Omega}|\nabla u|^{2} \mathrm{~d} x+\zeta(t) \int_{\Omega} u f(u) \mathrm{d} x \\
& =-2 E(u(t))+\zeta(t) \int_{\Omega}(u f(u)-F(u)) \mathrm{d} x,
\end{aligned}
$$

where $E(u(t))$ is given by (1.13). Taking advantage of (1.6), we obtain that

$$
y^{\prime}(t) \geq-2 E(u(t))+\frac{\epsilon C_{0}}{2+\epsilon} \zeta(t) \int_{\Omega}|u|^{\alpha} \mathrm{d} x
$$

Moreover, we compute

$$
E^{\prime}(u(t))=-\int_{\Omega} u_{t}^{2} \mathrm{~d} x-\zeta^{\prime}(t) \int_{\Omega} F(u) \mathrm{d} x \leq 0,
$$

thanks to (1.7). It then follows that $E(u(t))$ is non-decreasing in $t$ so that we have

$$
E(u(t)) \leq E(u(0))=E\left(u_{0}\right), \quad t \geq 0 .
$$

From (2.5), (2.7), and the Hölder inequality, we find that

$$
y^{\prime}(t) \geq-2 E\left(u_{0}\right)+\frac{\epsilon \zeta(0) C_{0} 2^{\alpha / 2}}{(2+\epsilon)|\Omega|^{\alpha / 2-1}} y(t)^{\alpha / 2}
$$

To conclude the proof we use the following result.

Lemma 2.1. Let $y:[0, T) \rightarrow[0, \infty)$ be a $C^{1}$-function satisfying

$$
y^{\prime}(t) \geq-C_{1}+C_{2} y(t)^{q}
$$

for some constants $C_{1} \in \mathbb{R}, C_{2}>0, q>1$. Then

$$
T \leq \begin{cases}\frac{y^{1-q}(0)}{C_{2}(q-1)} & \text { if } C_{1} \leq 0 \\ \int_{y(0)}^{\infty} \frac{d z}{C_{2} z^{q}-C_{1}} & \text { if } C_{1}>0 \text { and } y(0)>\left(\frac{C_{1}}{C_{2}}\right)^{1 / q}\end{cases}
$$


Proof of Lemma 2.1. We give the proof here for completeness. If $C_{1} \leq 0$ then $y^{\prime}(t) \geq C_{2} y(t)^{q}$. It follows that

$$
y^{\prime} y^{-q}=\frac{\mathrm{d}}{\mathrm{d} t}\left(\frac{y^{1-q}}{1-q}\right) \geq C_{2} .
$$

Integrating this differential inequality yields the desired upper bound in this case.

Suppose now that $C_{1}>0$ and $y(0)>\left(\frac{C_{1}}{C_{2}}\right)^{1 / q}$. Then $y(t)>\left(\frac{C_{1}}{C_{2}}\right)^{1 / q}$ for all $0 \leq t<T$. Therefore

$$
\frac{y^{\prime}(t)}{C_{2} y(t)^{q}-C_{1}} \geq 1, \quad 0 \leq t<T \text {. }
$$

Integrating this differential inequality yields

$$
t \leq \int_{0}^{t} \frac{y^{\prime}(\tau) \mathrm{d} \tau}{C_{2} y(\tau)^{q}-C_{1}} \leq \int_{y(0)}^{\infty} \frac{\mathrm{d} z}{C_{2} z^{q}-C_{1}}<\infty
$$

This finishes the proof of Lemma 2.1.

\subsection{Proof of Theorem 1.4}

We define

$$
H(t)=\zeta(t) \int_{\Omega} F\left((u(t, x)) \mathrm{d} x-\frac{1}{p} \int_{\Omega}|\nabla u(t, x)|^{p} \mathrm{~d} x,\right.
$$

and

$$
L(t)=\frac{1}{2}\|u(t)\|_{2}^{2}
$$

By using (1.1), we obtain that

$$
\begin{aligned}
H^{\prime}(t) & \left.=\int_{\Omega} u_{t}^{2}(t, x)\right) \mathrm{d} x+\zeta^{\prime}(t) \int_{\Omega} F(u(t, x)) \mathrm{d} x \\
& \left.=\frac{\zeta^{\prime}(t)}{\zeta(t)} H(t)+\int_{\Omega} u_{t}^{2}(t, x)\right) \mathrm{d} x+\frac{\zeta^{\prime}(t)}{p \zeta(t)} \int_{\Omega}|\nabla u(t, x)|^{p} \mathrm{~d} x \\
& \geq \frac{\zeta^{\prime}(t)}{\zeta(t)} H(t) .
\end{aligned}
$$

Hence $H(t) \geq H(0) \geq 0$, by virtue of (1.7).

Recalling (1.1), (2.11), and (1.15), we compute

$$
\begin{aligned}
L^{\prime}(t) & =-\int_{\Omega}|\nabla u(t, x)|^{p} \mathrm{~d} x+\zeta(t) \int_{\Omega} u(t, x) f(u(t, x)) \mathrm{d} x \\
& \geq-\int_{\Omega}|\nabla u(t, x)|^{p} \mathrm{~d} x+\kappa \zeta(t) \int_{\Omega} F(u(t, x)) \mathrm{d} x \\
& \geq \kappa H(t)+\left(\frac{\kappa}{p}-1\right) \int_{\Omega}|\nabla u(t, x)|^{p} \mathrm{~d} x
\end{aligned}
$$




$$
\geq\left(\frac{\kappa}{p}-1\right) \int_{\Omega}|\nabla u(t, x)|^{p} \mathrm{~d} x
$$

Applying Hölder inequality and then Poincaré inequality yields

$$
L(t) \leq|\Omega|^{1-2 / p}\left(\int_{\Omega}|u(t, x)|^{p} \mathrm{~d} x\right)^{2 / p} \leq C\left(\int_{\Omega}|\nabla u(t, x)|^{p} \mathrm{~d} x\right)^{2 / p},
$$

where $C>0$ is a constant depending only on $\Omega$ and $p$. Hence

$$
L^{\prime}(t) \geq \frac{\kappa-p}{p C^{p / 2}} L^{p / 2}(t)
$$

Integrating the differential inequality (2.13) leads to

$$
t \leq \frac{2 p C^{p / 2} L^{1-p / 2}(0)}{(p-2)(\kappa-p)}<\infty .
$$

Therefore $u$ blows up at a finite time $T^{*} \leq \frac{2 p C^{p / 2} L^{1-p / 2}(0)}{(p-2)(\kappa-p)}$.

\subsection{Proof of Proposition 1.1}

Recalling (1.15), we obtain that

$$
F(u) \geq C u^{\kappa} \text { for all } u \geq 1
$$

for some constant $C>0$. Let $K \subset \Omega$ be a compact nonempty subset of $\Omega$. Fix a smooth cut-of function $\phi \in C^{\infty}(\Omega)$ such that

$$
\phi(x)=1 \quad \text { for } x \in K .
$$

We look for initial data $u_{0}=\lambda \phi$ where $\lambda>0$ to be chosen later. Clearly $u_{0} \in L^{\infty}(\Omega) \cap$ $W_{0}^{1, p}(\Omega)$, and for $\lambda \geq 1$ we have using (2.14)

$$
\begin{aligned}
\mathbf{E}_{p}\left(u_{0}\right) & =\frac{1}{p} \int_{\Omega}\left|\nabla u_{0}\right|^{p}-\zeta(0) \int_{\Omega} F\left(u_{0}\right), \\
& =\frac{\lambda^{p}}{p} \int_{\Omega}|\nabla \phi|^{p}-\zeta(0) \int_{K} F(\lambda)-\zeta(0) \int_{\Omega \backslash K} F\left(u_{0}\right), \\
& \leq \frac{\lambda^{p}}{p} \int_{\Omega}|\nabla \phi|^{p}-\tilde{C} \lambda^{\kappa},
\end{aligned}
$$

for some constant $\tilde{C}>0$. Since

$$
\frac{\lambda^{p}}{p} \int_{\Omega}|\nabla \phi|^{p}-\tilde{C} \lambda^{\kappa} \leq 0 \text { for } \lambda \geq\left(\frac{\|\nabla \phi\|_{p}^{p}}{p \tilde{C}}\right)^{1 /(\kappa-p)},
$$

we deduce that $u_{0} \in \mathcal{E}$ for $\lambda$ large enough. This finishes the proof of Proposition 1.1. 


\section{Acknowledgement}

The authors are grateful to the anonymous referee for a careful reading of the manuscript and for his/her constructive comments.

\section{References}

[1] Quittner P., Souplet P., Superlinear Parabolic Problems, Birkhäuser Verlag, Basel, xii+584, 2007.

[2] Straughan B., Explosive Instabilities in Mechanics, Springer, 1998.

[3] Bandle C., Brunner H., Blow-up in diffusion equations: a survey. J. Comput. Appl. Math., 97 (1998), 3-22.

[4] Payne L. E., Schaefer P. W., Lower bound for blow-up time in parabolic problems under Neumann conditions. Appl. Anal., 85 (2006), 1301-1311.

[5] Payne L. E., Philippin G. A., Blow-up phenomena in parabolic problems with time dependent coefficients under Dirichlet boundary conditions. Proc. Amer. Math. Soc., 141 (2013), 2309-2318.

[6] Payne L. E., Philippin G. A., Blow-up phenomena for a class of parabolic systems with timedependent coefficients. Applied Mathematics, 3 (2012), 325-330.

[7] Ding J., Shen X., Blow-up in $p$-Laplacian heat equations with nonlinear boundary conditions. Z. Angew. Math. Phys., 67, Article number: 125 (2016).

[8] Liao M., Liu Q. and Ye H., Global existence and blow-up of weak solutions for a class of fractional $p$-Laplacian evolution equations. Adv. Nonlinear Anal., 9 (2020), 1569-1591.

[9] Liu W., Wang M., Blow-up of the solution for a $p$-Laplacian equation with positive initial energy. Acta Appl. Math., 103 (2008), 141-146.

[10] Messaoudi S. A., A note on blow up of solutions of a quasilinear heat equation with vanishing initial energy. J. Math. Anal. Appl., 273 (2002), 243-247.

[11] Levine H. A., Some nonexistence and instability theorems for solutions of formally parabolic equations of the form $\mathcal{P} u_{t}=-\mathcal{A} u+\mathcal{F}(u)$. Arch. Ration. Mech. Anal., 51 (1973), 371-386.

[12] Kaplan S., On the growth of solutions of quasi-linear parabolic equations. Comm. Pure Appl. Math., 16 (1963), 305-330.

[13] Galaktionov V. A., Pohozaev S. I., Existence and blow-up for higher-order semilinear parabolic equations: majorizing order-preserving operators. Indiana Univ. Math. J., 51 (2002), 1321-1338.

[14] Galaktionov V. A., Pohozaev S. I., Blow-up and critical exponents for nonlinear hyperbolic equations. Nonlinear Anal., 53 (2003), 453-466.

[15] Pucci P., Serrin J., Global nonexistence for abstract evolution equations with positive initial energy. J. Differential Equations, 150 (1998), 203-214.

[16] Junning Z., Existence and nonexistence of solutions for $u_{t}=\operatorname{div}\left(|\nabla u|^{p-2} \nabla u\right)+f(\nabla u, u, x, t)$. J. Math. Anal. Appl., 172 (1993), 130-146. 